\title{
Special Hperbolic Type Approximation for Solving of 3-D Two Layer Stationary Diffusion Problem
}

\author{
Ilmārs Kangro \\ Faculty of Engineering \\ Rezekne Academy of Technologies \\ Rezekne, Latvia \\ ilmars.kangro@rta.lv \\ Edmunds Teirumnieks \\ Faculty of Engineering \\ Rezekne Academy of Technologies \\ Rezekne, Latvia \\ Edmunds.Teirumnieks@rta.lv
}

\author{
Harijs Kalis \\ Institute of Mathematics and Computer \\ sciences \\ University of Latvia \\ Riga, Latvia \\ harijs.kalis@lanet.lv
}

\author{
Ërika Teirumnieka \\ Faculty of Engineering \\ Rezekne Academy of Technologies \\ Rezekne, Latvia \\ Erika.Teirumnieka@rta.lv
}

\begin{abstract}
In this paper we examine the conservative averaging method (CAM) along the vertical z-coordinate for solving the 3-D boundary-value 2 layers diffusion problem. The special parabolic and hyperbolic type approximation (splines), that interpolate the middle integral values of piece-wise smooth function, is investigated. With the help of these splines the problems of mathematical physics in 3-D with respect to one coordinate are reduced to problems for system of equations in 2-D in every layer. This procedure allows reduce also the 2-D problem to a 1-D problem and the solution of the approximated problem can be obtained analytically. As the practical application of the created mathematical model, we are studying the calculation of the concentration of heavy metal calcium (Ca) in a two-layer peat block.
\end{abstract}

Keywords-conservative averaging method, finitedifference method, diffusion problem, special splines.

\section{INTRODUCTION}

The boundary value problems (BVP) described by PDE with piece-wise coefficients in multi-layered domains are currently the subject of studies [4]. The interest is often caused by problems in itself, but even more interesting are their solutions: mainly numerical ones, because analytical solutions can only be obtained in the ordinary sense (without changing the number of dimensions of the boundary-value problem) in the simplest cases [2]. The article deals with a universal method for solving the second order of partial differential equations - a consider conservative averaging method (CAM), the essence of which is a reduction in the number of dimensions of a given BVP, with a view to obtaining analytical expressions (formulas) of the solution. The further solution of the BVP includes a repeated reduction in the number of dimensions or applying of numerical methods to solve the acquired BVP. The unknown function is replaced by the approximated solution - the special spline with two different functions, which interpolate the middle integral values of piece-wise smooth function. The functions of the hyperbolic type spline are created and used with parameters that have to be chosen in the appropriate way to decrease the error of approximation of the solution. It should be noted that, in limit case when the parameters of spline function tends to zero we have the integral parabolic spline, obtained from A.Buikis [3]. The 2-D boundary value problem obtained by the conservative averaging method (CAM) was solved numerically using the finite-difference method in the case of parabolic and hyperbolic splines. A test example, a solution to the given 3-D boundary value problem, was created for numerical approbation of the averaging method, where the unknown function was a solution to the corresponding 1-D boundary value problem for two ODEs. The solution of ODEs' could be obtained both analytically (exact solution) and numerically by the averaging method using parabolic type and hyperbolic type splines. This in turn allowed a comparison of the analytical solution with the obtained numerical solutions.

\section{Materials AND METHODS}

\section{The Mathematical Model}

The process of diffusion is considered in 3-D parallelepiped

$$
\Omega=\{(x, y, z): 0 \leq x \leq l, 0 \leq y \leq L, 0 \leq z \leq Z\}
$$

. The domain $\Omega$ consists of two layer medium. We will consider the stationary 3-D problem of the linear diffusion theory for multilayered piece-wise homogenous materials of $N$ layers in the form $\Omega_{i}=\left\{(x, y, z): x \in(0, l) \quad y \in(0, L) \quad z \in\left(z_{i-1}, x_{i}\right)\right\}, i=\overline{1, N}$ where $H_{i}=z_{i}-z_{i-1}$ is the height of layer 
$\Omega_{i}, z_{0}=0, z_{N}=Z$. The distribution of concentrations $c_{i}=c_{i}(x, y, z)$ in every layer $\Omega_{i}$ at the point $(x, y, z)=\Omega_{i}$ should be calculated by solving the following partial differential equation(PDE):

$$
\begin{aligned}
& D_{i x} \partial^{2} c_{i} / \partial x^{2}+D_{i y} \partial^{2} c_{i} / \partial y^{2}+D_{i z} \partial^{2} c_{i} / \partial z^{2}+ \\
& f_{i}(x, y, z)=0
\end{aligned}
$$

$D_{i x}, D_{i y}, D_{i z}$ are constant diffusion coefficients, $c_{i}=c_{i}(x, y, z)$ - the concentrations functions in every layer, $f_{i}(x, y, z)$ - the fixed sources functions. The values $c_{i}$ and the flux functions $D_{i z} \partial c_{i} / \partial z$ must be continuous on the contact lines between the layers $z=z_{i}, \overline{i=1, N-1}$ :

$\left.c_{i}\right|_{z_{i}}=\left.c_{i+1}\right|_{z_{i}}, \quad D_{\dot{z}} \partial c_{i} /\left.\partial z\right|_{z_{i}}=D(i+1) z \partial c(i+1) /\left.\partial z\right|_{z_{i}}$

where $\overline{i=1, N-1}$. The layered material is bounded above and below with the plane surfaces $z=0, z=Z$ with fixed boundary conditions in following form:

$$
D_{1 z} \partial c_{1}(x, y, 0) / \partial z=0, \quad c_{N}(x, y, z)=C_{a}(x, y),
$$

or $c_{1}(x, y, 0)=C_{0}(x, y), \quad c_{N}(x, y, Z)=C_{a}(x, y)$, tions.

$C_{0}(x, y), C_{a}(x, y)$ are the given concentration-func-

We have two forms of fixed boundary conditions in the $x, y$ directions: 1) The periodical conditions by $x=0, x=l(1.5)$ :

$c_{i}(0, y, z)=c_{i}(l, y, z), \partial c_{i}(0, y, z) / \partial x=\partial c_{i}(l, y, z) / \partial x$,

2) The symmetrical conditions by $y=0, y=L$ $\partial c_{i}(x, 0, z) / \partial y=\partial c_{i}(x, L, z) / \partial y=0$.

We will use the CAM and the finite difference (FD) method to solve the problem (1.1)-(1.6). These procedures allow reduce the 3-D problem to some 2-D boundary-value problem (BVP) for the system of partial differential equations with circular matrix in the $x$-direction.

2.The conservative averaging method with parabolic splines

The equation of (1.1) are averaged along the heights $H_{i}$ of layers $\Omega_{i}$ and quadratic integral splines along $z$ coordinate in the following form one used [3]

$c_{i}(x, y, z)=C_{i}(x, y)+m_{i}(x, y)\left(z-\bar{z}_{i}\right)+$

$e_{i}(x, y) G_{i}\left(\left(z-\bar{z}_{i}\right)^{2} / H_{i}^{2}-1 / 12\right)$

$G_{i}=H_{i} / D_{i z}, \bar{z}_{i}=\left(z_{i-1}+z_{i}\right) / 2 . m_{i}, e_{i}, C_{i}$ - the unknown coefficients of the spline-function,

$$
C_{i}(x, y)=H_{i}^{-1} \int_{z_{i-1}}^{z_{i}} c_{i}(x, y, z) d z \text { - the average }
$$

values of $c_{i}, i=\overline{1, N}$.

After averaging the system (1.1) along every layer $\Omega_{i}$ we obtain

$$
\begin{aligned}
& D_{i x} \partial^{2} C_{i} / \partial x^{2}+D_{i y} \partial^{2} C_{i} / \partial y^{2}+2 H_{i}^{-1} e_{i}+, \\
& F_{i}(x, y)=0
\end{aligned}
$$

$C_{i}(x, y)=H_{i}^{-1} \int_{z_{i-1}}^{z_{i}} c_{i}(x, y, z) d z$ - the average values of $f_{i}, i=\overline{1, N}$. From (1.1), (2.1) using boundary conditions (1.3) we can determine the unknown functions $m_{i}, e_{i}$ . Therefore, from (2.2) we obtain the system of $N$ partial differential equations (PDE), where the boundary conditions for $C_{i}$ are determined from (1.4)-(1.5) in the $x, y$-directions for averaged values

$C_{i}(0, y)=C_{i}(l, y), \partial C_{i}(0, y) / \partial x=\partial C_{i}(l, y) / \partial x$

$\partial C_{i}(x, 0) / \partial y=\partial C_{i}(x, L) / \partial y=0$.

In the case $N=2$ (two layers) we have (2.5):

$$
\begin{aligned}
& e_{1}=\left(9 C_{2}-(12+3 / k) C_{1}-3 C_{a}+(6+3 / k) C_{0}\right) /, \\
& \left(2 G_{2}+2 G_{1}\right) \\
& e_{2}=\left(9 C_{1}-(12+3 k) C_{2}+(6+3 k) C_{a}-3 C_{0}\right) /, \\
& \left(2 G_{2}+2 G_{1}\right) \\
& k=G_{1} / G_{2} .
\end{aligned}
$$

We have from (2.2) the following system of two PDE $\left\{\begin{array}{l}D_{1 x} \partial^{2} C_{1}(x, y) / \partial x^{2}+D_{1 y} \partial^{2} C_{1}(x, y) / \partial y^{2}+ \\ 2 H_{1}^{-1} e_{1}(x, y)+F_{1}(x, y)=0, \\ D_{2 x} \partial^{2} C_{2}(x, y) / \partial x^{2}+D_{2 y} \partial^{2} C_{2}(x, y) / \partial y^{2}+ \\ 2 H_{2}^{-1} e_{2}(x, y)+F_{2}(x, y)=0 .\end{array}\right.$

After resolving (2.6), the concentration functions $c_{i}=c_{i}(x, y, z)$ shall be obtained

$c_{1}(x, y, z)=C_{1}(x, y)+m_{1}(x, y)\left(z-H_{1} / 2\right)+$ $e_{1}(x, y) G_{1}\left(\left(z-H_{1} / 2\right)^{2} / H_{1}^{2}-1 / 12\right), z \in\left[0, H_{1}\right] "$ $c_{2}(x, y, z)=C_{2}(x, y)+m_{2}(x, y)\left(z-\left(2 H_{1}+H_{2}\right) / 2\right)+$ $e_{2}(x, y) G_{2}\left(\left(z-\left(2 H_{1}+H_{2}\right) / 2\right)^{2} / H_{2}^{2}-1 / 12\right), z \in\left[H_{1}, Z\right]^{\cdot}$

From (1.3) it is obtained

$m_{1}(x, y)=e_{1}(x, y) /\left(3 D_{1 z}\right)+2\left(C_{1}(x, y)-C_{0}(x, y)\right) / H_{1}$, $m_{2}(x, y)=-e_{2}(x, y) /\left(3 D_{2 z}\right)+2\left(C_{a}(x, y)-C_{2}(x, y)\right) / H_{2}$

3.The conservative averaging method with hyperbolic type splines in 2 layers

The equation of (1.1) is averaged along the heights $H_{i}$ of layers $\Omega_{i}$ using the hyperbolic type splines. Applying averaged method with respect to $z$ we use the approximate solution with two fixed parametrical functions $f_{i 1}, f_{i 2}, i=\overline{1,2}$ 
$c_{i}(x, y, z)=C_{i}(x, y)+m_{i}(x, y) f_{i 1}\left(z-\bar{z}_{i}\right)+$

$e_{i}(x, y)+f_{i 2}\left(z-\bar{z}_{i}\right)$

$C_{i}(x, y)=H_{i}^{-1} \int_{z_{i-1}}^{z_{i}} c_{i}(x, y, z) d z$ - the averaged values,

according to the definition of the spline function

$\int_{z_{i-1}}^{z_{i}} f_{i 1}(z) d z=\int_{z_{i-1}}^{z_{i}} f_{i 2}(z) d z=0$

$\bar{z}_{i}=\left(z_{i-1}+z_{i}\right) / 2, z \in\left[z_{i-1}, z_{i}\right]$,

$f_{i z 1}=\frac{0.5 H_{i} \sinh \left(a_{i}\left(z-\bar{z}_{i}\right)\right)}{\sinh \left(0.5 a_{i} H_{i}\right)}$

$f_{i z 2}=\frac{\cosh \left(a_{i}\left(z-\bar{z}_{i}\right)\right)-A_{i}}{8 \sinh ^{2}\left(0.5 a_{i} H_{i}\right)}$

$A_{i}=\frac{0.5 \sinh \left(0.5 a_{i} H_{i}\right)}{a_{i} L / 2}, i=\overline{1,2}$.

$a_{i}>0$ are fixed parameters (unknown). It should be noted if parameters $a_{i}$ tend to zero then the integral parabolic spline from [3] is obtained in the limit case. The unknown functions $m_{i}(x, y) e_{i}(x, y)$ we can determined from boundary conditions at $z=0, z=Z$ :

$$
\begin{aligned}
& d_{i z}=0.5 H_{i} a_{i} \operatorname{coth}\left(0.5 a_{i} H_{i}\right) \\
& k_{i z}=0.25 a_{i} \operatorname{coth}\left(0.25 a_{i} H_{i}\right) \\
& C_{1}-0.5 m_{1} H_{1}+e_{1} b_{1 z}=C_{0} \\
& C_{2}+0.5 m_{2} H_{2}+e_{2} b_{2 z}=C_{a} \\
& b_{i z}=\frac{\cosh \left(0.5 a_{i} H_{i}\right)-A_{i}}{8 \sinh ^{2}\left(0.25 a_{i} H_{i}\right)} \\
& D_{1 z}\left(m_{1} d_{1 z}+e_{1} k_{1 z}\right)=D_{2 z}\left(m_{2} d_{2 z}-e_{2} k_{2 z}\right) \\
& C_{1}+0.5 m_{1} H_{1}+e_{1} b_{1 z}=C_{2}-0.5 m_{2} H_{2}+e_{2} b_{2 z} \\
& m_{1}=2\left(C_{1}+b_{1 z} e_{1}\right) / H_{1} .
\end{aligned}
$$

Thus we have a system of 2 algebraic equations for determining $e_{i}, i=\overline{1,2}$ :

$$
\begin{aligned}
& b_{11} e_{1}+b_{12} e_{2}=b_{3} C_{a}+b_{4} C_{0}-b_{3} C_{2}-b_{4} C_{1} \\
& b_{21} e_{1}+b_{22} e_{2}=2 C_{2}-2 C_{1}-C_{a}+C_{0}, \text { where } \\
& b_{11}=b_{4} b_{1 z}+k_{1 z}, b_{12}=b_{3} b_{2 z}+D_{21} k_{2 z}, b_{21}=2 b_{1 z} \\
& b_{22}=-2 b_{2 z}, b_{3}=2 D_{21} d_{2 z} / H_{2}, b_{4}=2 d_{1 z} / H_{1}, \\
& D_{21}=D_{2 z} / D_{1 z} .
\end{aligned}
$$

The solution for $e_{1}, e_{2}$ is:

$$
\begin{gathered}
e_{1}=b_{17} C_{1}+b_{18} C_{2}+d_{4} C_{a}+d_{6} C_{0} \\
, \quad e_{2}=b_{27} C_{1}+b_{28} C_{2}+d_{5} C_{a}+d_{7} C_{0}, \text { where } \\
b_{17}=\left(-b_{4} b_{22}+2 b_{12}\right) / \operatorname{det}, \quad b_{18}=\left(-b_{3} b_{22}-2 b_{12}\right) / \operatorname{det} \\
, \quad b_{27}=\left(b_{4} b_{21}-2 b_{11}\right) / \operatorname{det}, b_{28}=\left(b_{3} b_{21}+2 b_{11}\right) / \operatorname{det}
\end{gathered}
$$

$$
\begin{aligned}
& d_{4}=\left(b_{3} b_{22}+b_{12}\right) / \operatorname{det}, \quad d_{6}=\left(b_{4} b_{22}-b_{12}\right) / \operatorname{det}, \\
& d_{5}=-\left(b_{3} b_{21}+b_{11}\right) / \operatorname{det}, \quad d_{7}=\left(-b_{4} b_{21}+b_{11}\right) / \operatorname{det}, \\
& \operatorname{det}=b_{11} b_{22}-b_{21} b_{12} .
\end{aligned}
$$

The 2-D boundary-value problem is in following form:

$$
\left\{\begin{array}{l}
\frac{\partial}{\partial x}\left(D_{1 x} \frac{\partial C_{1}}{\partial x}\right)+\frac{\partial}{\partial y}\left(D_{1 y} \frac{\partial C_{1}}{\partial y}\right)+b_{5} e_{1}+F_{1}=0 \\
\frac{\partial}{\partial x}\left(D_{2 x} \frac{\partial C_{2}}{\partial x}\right)+\frac{\partial}{\partial y}\left(D_{2 y} \frac{\partial C_{2}}{\partial y}\right)+b_{6} e_{2}+F_{2}=0 \\
\frac{\partial c_{i z}(0, y)}{\partial x}=\frac{\partial c_{i z}(x, 0)}{\partial y}=0, c_{i z}(l, y)=c_{i z}(x, L)=0,
\end{array}\right.
$$

where $b_{5}=2 D_{1 z} k_{1 z} / H_{1}, b_{6}=2 D_{2 z} k_{2 z} / H_{2}$.

\section{The finite-difference method for two layers with parabolic type splines}

We consider an uniform grid $\left(N_{x} \times\left(N_{y}+1\right)\right)$ [7],

$\omega_{h}=\left\{\begin{array}{l}\left(x_{i}, y_{j}\right), x_{i}=h_{x}, y_{j}=(j-1) h_{y}, i=\overline{1, N_{x}}, \\ j=\overline{1, N_{y}+1}, N_{x} h_{x}=l, N_{y} h_{y}=L\end{array}\right\}$.

Subscripts $(i, j)$ refer to $x, y$ indices; the mesh spacing in the $x_{i}, y_{j}$ directions is $h_{x}, h_{y}$. For two layers ( $N=2$ ) we can the PDEs (2.6) rewritten in following vector form:

$$
D_{x} \partial^{2} C / \partial x^{2}+D_{y} \partial^{2} C / \partial y^{2}-A C+F=0,
$$

where $D_{x}, D_{y}$ are the 2 order diagonal matrices with elements $D_{1 x}, D_{2 x}$ and $D_{1 y}, D_{2 y} . A$ is the matrix of second order, $C$ is the 2 nd order vectors-column with elements $C_{1}, C_{2}$ and $F$ is the 2 order vectors-column with following elements :

$$
\begin{gathered}
\left(\begin{array}{c}
F_{1}-\left(\left(6+3 k^{-1}\right) C_{0}-3 C_{a}\right) /\left(H_{1} d_{1}\right) \\
F_{1}+\left((6+3 k) C_{a}-3 C_{0}\right) /\left(H_{2} d_{1}\right)
\end{array}\right)^{T}, \\
A=\frac{1}{d_{1}}\left(\begin{array}{cc}
\left(12+3 k^{-1}\right) / H_{1} & -9 / H_{1} \\
-9 / H_{2} & (12+3 k) / H_{2}
\end{array}\right),
\end{gathered}
$$

$\left(d_{1}=G_{2}+G_{1}\right)$. The equation (4.1) with periodical conditions (2.4) for vector function $C$ in the uniform grid $\left(x_{i}, y_{j}\right)$ is replaced by vector difference equations of second order approximation in 3-point stencil [1]:

$$
A \quad W_{j-1}-C W_{j}+B \quad W_{j+1}+F_{j}=0,
$$

where $W_{j}$ are vectors-column

$$
\left(W_{j} \approx\left(C_{1, j}, C_{2, j}, \ldots, C_{N_{x}, j}\right)^{T}\right), F_{j} \text { are vectors-column }
$$

with elements $\left(F_{1, j}, F_{2, j}, \ldots, F_{N_{x}, j}\right)^{T}, \overline{j=2, N_{y}}$

, $A A, C C, B B=A A$ are the block- matrices of second 
order with the elements of the circular symmetric matrix with $N_{x}=M$-order in the following form (it is possible to define the circular matrix with the first row that is in the form $\left.A=\left\lfloor a_{1}, a_{2}, \ldots, a_{M}\right\rfloor\right)$ :

$$
\begin{aligned}
A & \left.=\left(\begin{array}{c}
{\left[D_{1 y} / h_{y}^{2}, 0, \ldots, 0\right.} \\
0
\end{array}\right]\left[\begin{array}{c}
0 \\
D_{2 y} / h_{y}^{2}, 0, \ldots, 0
\end{array}\right]\right), \\
C & =\left(\begin{array}{lll}
c_{1} & c_{2} \\
c_{3} & c_{4}
\end{array}\right),
\end{aligned}
$$

where the circular matrices $c_{1}, c_{2}, c_{3}, c_{4}$ are

$$
\begin{aligned}
c c_{1}=\left[\begin{array}{l}
2\left(D_{1 x} / h_{x}^{2}+D_{1 y} / h_{y}^{2}\right)+\left(12+3 k^{-1}\right) /\left(H_{1} d_{1}\right),-D_{1 x} / h_{x}^{2}, \\
0, \ldots, 0,-D_{1 x} / h_{x}^{2}
\end{array}\right] \\
c c_{2}=\left[-9 /\left(H_{1} d_{1}\right), 0, \ldots, 0\right], \quad c c_{3}=\left[-9 /\left(H_{2} d_{1}\right), 0, \ldots, 0\right], \\
c c_{4}=\left[\begin{array}{l}
\left.2\left(D_{2 x} / h_{x}^{2}+D_{2 y} / h_{y}^{2}\right)+(12+3 k) /\left(H_{2} d_{1}\right),\right] . \\
-D_{2 x} / h_{x}^{2}, 0, \ldots, 0,-D_{2 x} / h_{x}^{2}
\end{array}\right] .
\end{aligned}
$$

The boundary conditions (2.4) are replaced by difference equations of first order approximation:

$$
C\left(x, h_{y}\right)=C(x, 0)+O\left(h_{y}^{2}\right), \quad C(x, L)=C\left(x, L-h_{y}\right)+O\left(h_{y}^{2}\right) .
$$

5.The finite-difference method for two layers with hyperbolic type splines

The vector $F$ and matrix $A$ in (4.1) are

$F_{1}+b_{5} d_{4} C_{a}+b_{5} d_{6} C_{0}, F_{2}+b_{6} d_{5} C_{a}+b_{6} d_{7} C_{0}$,

$A=\left(\begin{array}{ll}a_{11} & a_{12} \\ a_{21} & a_{22}\end{array}\right)$, where

$a_{11}=-b_{5} b_{17}, a_{12}=-b_{5} b_{18}, a_{21}=-b_{6} b_{27}$,

$a_{22}=-b_{6} b_{28}$.

The circular matrices $c_{i}$ are $a_{22}=-b_{6} b_{28}$

$$
\begin{aligned}
& c c_{1}=\left[\begin{array}{l}
2\left(D_{1 x} / h_{x}^{2}+D_{1 y} / h_{y}^{2}\right)+a_{11}, \\
-D_{1 x} / h_{x}^{2}, 0, \ldots, 0,-D_{1 x} / h_{x}^{2}
\end{array}\right], \\
& c c_{2}=\left[a_{12}, 0, \ldots, 0\right], \quad c c_{3}=\left[a_{21}, 0, \ldots, 0\right], \\
& c c_{4}=\left[\begin{array}{l}
2\left(D_{2 x} / h_{x}^{2}+D_{2 y} / h_{y}^{2}\right)+a_{22}, \\
-D_{2 x} / h_{x}^{2}, 0, \ldots, 0,-D_{2 x} / h_{x}^{2}
\end{array}\right] .
\end{aligned}
$$

We use $a_{1}=15, a_{2}=13$.

\section{The numerical methods}

The vectors-column $W_{j}$ from (4.2) is calculated on Thomas algorithm [9] in the matrix form using MATLAB.

$$
W_{j}=X_{j} W_{j+1}+Y_{j}=0, j=N_{y}(-1) 1,
$$

where $X_{j}, Y_{j}$ are corresponding matrices and vectors, obtaining of following expressions (6.2):

$X_{j}=\left(C_{j}-A_{j} X_{j-1}\right)^{-1} B_{j}$,

$Y_{j}=\left(C_{j}-A_{j} X_{j-1}\right)^{-1}\left(A_{j} Y_{j}+F_{j}\right), \quad j=2(1) N_{y}$

Here $X_{1}=E, \mathrm{v}, W_{\bar{N}+1}=\left(E-X_{\bar{N}}\right)^{-1} Y_{\bar{N}},\left(\bar{N}=N_{y}\right)$,

where $E=\left(\begin{array}{cc}{[1,0, \ldots, 0]} & 0 \\ 0 & {[1,0, \ldots, 0]}\end{array}\right)$.

The inverse matrix of $A=\left(\begin{array}{cc}A_{1} & A_{2} \\ A_{3} & A_{4}\end{array}\right)$ is $B=A^{-1},(B A=A B=E), \quad B=\left(\begin{array}{ll}B_{1} & B_{2} \\ B_{3} & B_{4}\end{array}\right), \quad$ where $B_{4}=\left(A_{4}-A_{3} A_{1}^{-1} A_{2}\right)^{-1}, \quad B_{2}=-A_{1}^{-1} A_{2} B_{4}$, $B_{1}=\left(A_{1}-A_{2} A_{4}^{-1} A_{3}\right)^{-1}, B_{3}=-A_{4}^{-1} A_{3} B_{1}$.

The others operations with circular matrices and vectors of the second order can be easy obtain [6].

\section{Results and discussion}

\section{Approbation of numerical algorithms}

The special solution in the form $c_{1}(x, y, z)=g_{1}(z) \cos (\pi y / L) \sin (2 \pi x / l)$

$c_{2}(x, y, z)=g_{2}(z) \cos (\pi y / L) \sin (2 \pi x / l)$ of the PDE

(1.1) was designed, where functions $g_{1}(z) g_{2}(z)$ was the solution of the following boundary value problem for two ODE (for boundary condition (1.3)):

$$
\begin{aligned}
& g_{1}^{\prime \prime}(z)-a_{1}^{2} g_{1}(z)=0, g_{1}(0)=0, g_{2}^{\prime \prime}(z)-a_{2}^{2} g_{2}(z)=0 \\
& , g_{2}(Z)=1, g_{1}\left(H_{1}\right)=g_{2}\left(H_{1}\right) \\
& D_{1 z} g_{1}^{\prime}\left(H_{1}\right)=D_{2 z} g_{2}^{\prime}\left(H_{1}\right), \text { where } \\
& a_{1}=\pi \sqrt{\left(\frac{4 D_{1 x}}{l^{2}}+\frac{D_{1 y}}{L^{2}}\right) / D_{1 z}} \\
& a_{2}=\pi \sqrt{\left(\frac{4 D_{2 x}}{l^{2}}+\frac{D_{2 y}}{L^{2}}\right) / D_{2 z}}
\end{aligned}
$$

The analytical solution of boundary-value problem of ODEs (7.1) is [5]:

$$
g_{1}(z)=P_{1} \sinh \left(a_{1} z\right) \text {, }
$$

$g_{2}(z)=P_{2} \cosh \left(a_{2} z\right)+P_{3} \sinh \left(a_{2} z\right)$, where the constants are:

$$
\begin{aligned}
& P_{1}=\frac{P_{2} \cosh \left(a_{2} H_{1}\right)+P_{3} \sinh \left(a_{2} H_{1}\right)}{\sinh \left(a_{1} H_{1}\right)} \\
& P_{2}=\frac{1-P_{3} \sinh \left(a_{2} Z\right)}{\cosh \left(a_{2} Z\right)},
\end{aligned}
$$


$P_{3}=\frac{\cosh \left(\left(a_{1}-a_{2}\right) H_{1}\right) a_{1} D_{1 z}}{\sinh \left(a_{2} H_{2}+a_{1} H_{1}\right) a_{2} D_{2 z}}+$ $\frac{\sinh \left(a_{1} H_{1}\right) \sinh \left(a_{2} H_{1}\right)\left(a_{1} D_{1 z}-a_{2} D_{2 z}\right)}{\sinh \left(a_{2} H_{2}\right) \cosh \left(a_{1} H_{1}\right)\left(a_{1} D_{1 z}-a_{2} D_{2 z}\right)}$

Based on the literature source [4], it can be proofed that the solution to the BVP (7.1), obtained by hyperbolic spline function, coincides with the analytical (exact) solution (7.2) of the BVP (7.1).

\section{Some numerical results}

Measurements of peat samples for the determination of heavy metals - iron and calcium concentrations were carried out in the swamp of the Vilani municipality Knavu swamp. Peat analyses have been performed with the OPTIMA 2100 MS ICP/OES Spectrometer of the inductively associated plasma optic emission spectrometer of the Perkin Elmer firm in the laboratories of the Geotechnology and Eco-Industrial Research Centre of Rezekne Academy of Technologies [8]. We consider the metal concentration in the 2 layered peat blocks $\Omega$ with following measure:

$L=l=1 \mathrm{~m}, H_{1}=1 \mathrm{~m}, H_{2}=1.5 \mathrm{~m}$,

$Z=H_{1}+H_{2}=2.5 m$.

On the top of the earth $(z=Z)$ we have the measured concentration $c[m g / k g] \times 1000$ of calcium (Ca) in the following points in the $(x, y)$ plane:

$$
\begin{aligned}
& c(0.1,0.2)=0.62, c(0.5,0.2)=0.57 \\
& c(0.9,0.2)=0.43, c(0.1,0.8)=0.51, \\
& c(0.5,0.5)=0.43, c(0.9,0.5)=0.64 \\
& c(0.1,0.5)=0.58, c(0.5,0.8)=0.44 \\
& c(0.9,0.8)=0.72 .
\end{aligned}
$$

This date are smoothing by $2 \mathrm{D}$ interpolation with MATLAB operator, using the spline function. We use following diffusion coefficients in the layers:

$$
D_{1 z}=10^{-3}, D_{2 z}=5 \cdot 10^{-4}, D_{1 x}=D_{2 x}=10^{-4},
$$

$D_{1 y}=D_{2 y}=10^{-5}$. We can see the distribution of concentration $c$ in the $(x, y)$ plane for $\mathrm{Ca}$ at $z=H_{1}$ for hyperbolic (Fig. 1) and parabolic (Fig. 2) spline.

We can see the distribution of concentration $c$ in the $(x, y)$ plane for $\mathrm{Ca}$ at $z=H_{1}$ for hyperbolic (Fig. 1) and parabolic (Fig. 2) spline, in the Fig. 3 - the averaged concentration $C_{2}$ within the second layer for hyperbolic spline, in the Fig. $4-$ concentration's $c$ curve $\mathrm{S}$ at $y=L / 2$ for hyperbolic spline.

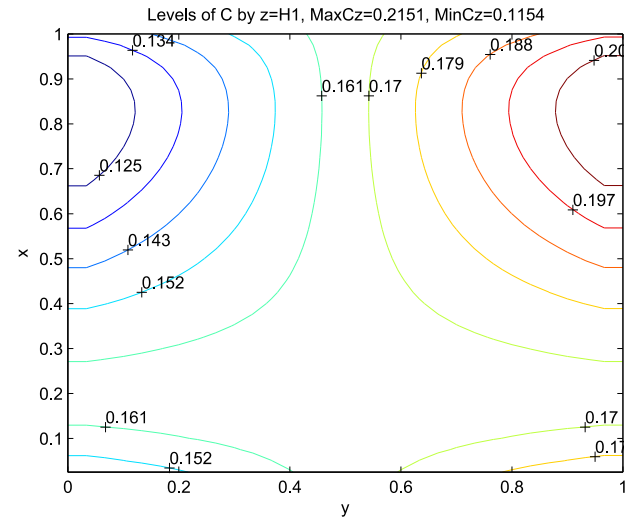

Fig. 1. Levels of $c$ at $z=H_{1}$ for hyperbolic spline.

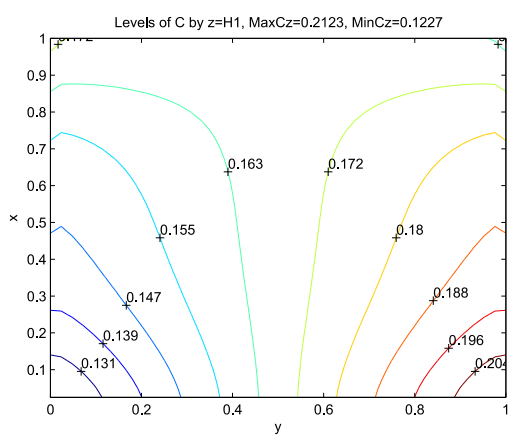

Fig. 2. Levels of $c$ at $z=H_{1}$ for parabolic spline.

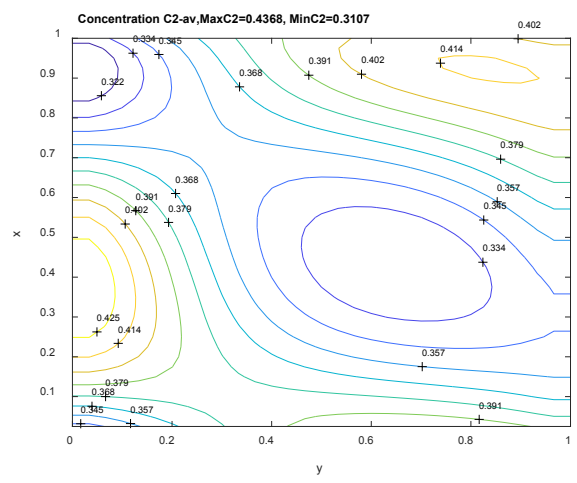

Fig. 3.The averaged concentration $C_{2}$ within the second layer for hyperbolic spline.

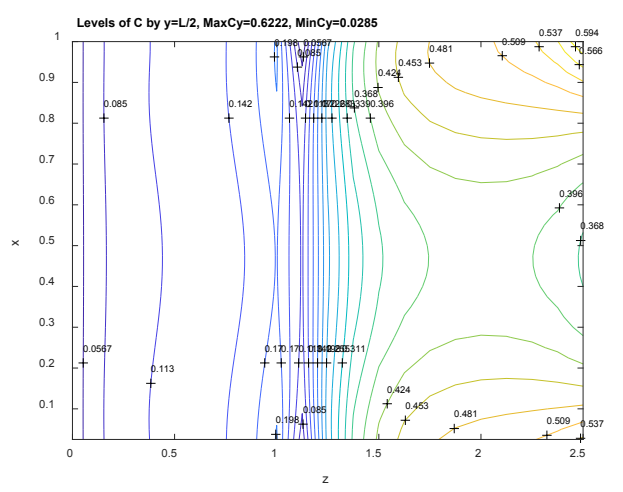

Fig. 4. Concentration's $c$ curve $\mathrm{s}^{\mathrm{s}}$ at $y=L / 2$ for hyperbolic spline. 


\section{Conclusions}

1. The 3D diffusion problem in $N$ layered domain described by a boundary value problem of the system of PDEs with piece-wise constant diffusion coefficients are approximated on the boundary value problem of a system of $N$ PDEs. The last mentioned system is solved due to the finite difference method.

2. For reducing the $3 \mathrm{D}$ diffusion problem to $2 \mathrm{D}$ boundary value problem of a system of PDEs the conservative averaging method (CAM) along the vertical z-coordinate by using parabolic type splines and newly designed special hyperbolic and type splines is studied.

3. The calculation process compared the effectiveness of the parabolic and hyperbolic splines usage for $3 \mathrm{D}$ diffusion problem reduction to $2 \mathrm{D}$ boundary value problem and resulted in higher accuracy of the approximated solution directly with hyperbolic type splines.

4. A Test example has been created allowed a comparison of the analytical (exact) solution of the 1-D BVP with the numerically obtained solutions of parabolic type splines and hyperbolic type splines, to assess their accuracy. It was found that the solution obtained by hyperbolic splines functions coincided with the analytical solution of the 1-D problem under consideration. This indicates the usefulness of the further usage of the CAM for solving BVP problems with the hepl of hyperbolic type splines compared to the previously widely used parabolic type splines.

5. The theoretical and practical problem studied makes it possible to obtain an engineering algorithm for mathematical modelling mass transfer processes in multilayered domain.

6. The results of the numerical experiments can give some new physical conclusions about the distribution of metals concentration in different layered peat blocks.

\section{References}

[1] .N. S. Bahvalov, N. P. Zhitkov and G. M. Kobelhkov, Numerical methods. M.: Nauka, 1987 (in Russian).

[2] J. Bear, Hydraulic of groundwater. Mc.Graw-Hill Inc., 1979.

[3] A. Buikis, "The approximation with splines for problems in layered systems," in Acta Universitatis Latviensis, Vol. 592, Riga, 1994, pp. 135-138 (in Latvian).

[4] A. Buikis, "The analysis of schemes for the modelling same processes of filtration in the underground, " in Acta Universitatis Latviensis, Vol. 592, Riga, 1994, pp. 25-32 (in Latvian).

[5] C. Henry Edwards and David E. Penny, Differential equations and boundary value problems, computing and modelling. Pearson, Prentice Hal, third edition, 2008 (in Russian).

[6] H. Kalis, A. Buiķis,A. Aboltins and I. Kangro, "Special Splines of Hyperbolic Type for the Solutions of Heat and Mass Transfer 3-D Problems in Porous Multi-Layered Axial Symmetry Domain," Mathematical Modelling and Analysis, vol. 22, issue 04, pp. 425-440, 2017.

[7] A. A. Samarskij, Theory of finite difference schemes. M.: Nauka, 1977 (in Russian).

[8] Ē. Teirumnieka, M., Kḷaviņš and E. Teirumnieks, "Major and trace elements in peat from bogs of east Latvia," in Mires and Peat /Ed. Māris Kḷlaviņšs, Riga: University of Latvia Press, 2010, pp. $115-124$.

[9] J. W.Thomas, Numerical partial differential equations. Finite difference methods. New-York: Springer-Verlag, Inc., 1995. 